\title{
Miller Fisher syndrome following Pfizer COVID-19 vaccine
}

\author{
Ana Abičić ${ }^{1}$ Ivan Adamec ${ }^{2}\left(\right.$ Mario Habek $^{2,3}$ \\ Received: 6 October 2021 / Accepted: 19 November 2021 / Published online: 24 November 2021 \\ (c) Fondazione Società Italiana di Neurologia 2021
}

\begin{abstract}
Introduction Miller Fisher syndrome (MFS) is a rare variant of Guillain-Barre syndrome characterized by ataxia, areflexia, and ophthalmoplegia. We present a case of MFS following Pfizer COVID-19 vaccine.

Case presentation A previously healthy 24-year-old female presented with binocular horizontal diplopia 18 days after receiving the first dose of Pfizer COVID-19 vaccine (Comirnaty $\left.{ }^{\circledR}\right)$. Anti-ganglioside testing revealed positive anti-GQ1b antibodies. Intravenous immunoglobulins were administered, in a dose of $2 \mathrm{~g}$ per $\mathrm{kg}$ of body weight over 5 days. On a follow-up exam 3 weeks after the treatment, clinical improvement was noted with normal bulbomotor examination.

Conclusion Patients with acute ophthalmoplegia occurring after COVID-19 vaccination should be screened for the presence of anti-GQ1b antibody. If the antibody is present, intravenous immunoglobulin should be administered as it may hasten clinical improvement.
\end{abstract}

Keywords COVID-19 $\cdot$ Pfizer vaccine $\cdot$ Miller Fisher syndrome

\section{Introduction}

Miller Fisher syndrome (MFS) is a rare variant of Guillain-Barre syndrome (GBS) characterized by ataxia, areflexia, and ophthalmoplegia [1]. Anti-GQ1b IgG antibody is detected in most patients with MFS, and studies have demonstrated that its titer reflects clinical severity scores, especially ophthalmoplegia [1]. MFS has been reported following infections and vaccinations with molecular mimicry being the likely mechanism triggering an immunological reaction [1]. During the pandemic of SARS-CoV-2 infection, several cases of MFS associated with COVID-19 have been described [2]. As more people gain access to COVID-19 vaccines, neurological complications following vaccination have also been reported. Waheed et al. described a case of

The adverse effect reported in this manuscript has been reported to Agency for Medicinal Products and Medical Devices of Croatia (HALMED)

Ivan Adamec

ivan.adamec@yahoo.com

1 General Hospital Zabok, Zabok, Croatia

2 Department of Neurology, University Hospital Center Zagreb, Referral Center for Autonomic Nervous System Disorders, Zagreb, Croatia

3 School of Medicine, University of Zagreb, Zagreb, Croatia
GBS following the first dose of Pfizer COVID-19 vaccine [3]. We present a case of MFS following Pfizer COVID-19 vaccine.

\section{Report of case}

A previously healthy 24-year-old female presented with binocular horizontal diplopia 18 days after receiving the first dose of Pfizer COVID-19 vaccine (Comirnaty®). Initially, in the $24 \mathrm{~h}$ following the vaccination, she experienced a bout of fever and diarrhea, symptoms that have been previously reported to occur after vaccination [4]. These symptoms resolved by the end of the first week post vaccination. Thirteen days after vaccination, the patient experienced itchy eyes, sneezing, and runny nose, which was attributed to allergic rhinitis and subsided completely on loratadine tablets. Eighteen days after vaccination with Pfizer COVID-19 vaccine, the patient developed double vision. Neurological examination in the emergency department revealed impaired abduction and elevation of both eyes, more prominent in the left eye (Video 1). The rest of the neurological examination was normal. Brain CT and CT angiography of head and neck were unremarkable. Routine laboratory examination was normal including complete blood count, blood glucose, creatinine, blood urea nitrogen, sodium, potassium, 
C-reactive protein, prothrombin time, and activated partial thromboplastin time. Intramuscular prostigmin test was negative. Brain and neck MRI were normal. Lumbar puncture was performed, and cerebrospinal fluid analysis showed albumin-cytological dissociation (albumin $296 \mathrm{mg} / \mathrm{L}$ (56-170), white blood count $2 \times 10^{6} / \mathrm{L}(<5)$. Intravenous methylprednisolone was administered, $500 \mathrm{mg}$ daily for 3 days with no improvement. Immunology tests revealed speckled ANA with positive anti-PCNA (proliferating cell nuclear antigen) antibodies. Other immunology tests were negative including rheumatoid factor, $\mathrm{C} 3, \mathrm{C} 4$, anti-dsDNA, anti-histone, anti-SS-A 52, anti-SS-A 60, anti-SS-B, antiSm, anti-U1-RNP, anti-Sm/RNP, anti-Scl-70, anti-Jo-1, antiCENP B, anti-ribosome, anti-PM-Scl, Acl IgG, Acl IgM, anti-beta2-GPI IgG, and anti-MAG. Anti-ganglioside testing revealed positive anti-GQ1b antibodies, with negative anti-GD1a, anti-GM1, anti-GM2, and anti-GD1b. Electromyoneurography was normal. These results were consistent with a limited form of MFS as there were no clinical signs of ataxia and neuropathy. Intravenous immunoglobulins (IVIG) were administered, in a dose of $120 \mathrm{~g}(2 \mathrm{~g}$ per $\mathrm{kg}$ of body weight) over 5 days. On a follow-up exam 3 weeks after the treatment, clinical improvement was noted with normal bulbomotor examination (Video 2). The patient reported double vision only in the far right and left eye position.

\section{Discussion}

The typical presentation of MFS consists of acute ophthalmoplegia with ataxia and areflexia. Anti-GQ1b IgG antibody is present in over $90 \%$ of MFS patients, decays rapidly with clinical recovery, and is not found in normal and disease control serum samples [1]. Acute ophthalmoplegia without ataxia, as in our patient, is an incomplete form of MFS associated with anti-GQ1b antibody [5]. The clinical spectrum of the anti-GQ1b antibody syndrome includes MFS, GBS, BBS (Bickerstaff's brainstem encephalitis) and acute ophthalmoplegia without ataxia. A study by Odaka et al. reported that in 110 patients with MFS and external ophthalmoplegia, tendon reflexes were found to be decreased or absent in 53\% [6]. Isolated abducens nerve palsy, without ataxia and signs of peripheral neuropathy, was suggested to be a mild form of MFS in a study by Tatsumoto et al. [7]. Therefore, the presentation of MFS solely with bulbomotor dysfunction, as was the case in the reported patient, is not uncommon.

The ganglioside GQ1b is abundant in cranial nerves supplying extraocular muscles and the presynaptic neuromuscular junction, which is devoid of a blood-nerve barrier [1]. There is in vitro evidence that anti-GQ1b IgG antibodies have pathogenic effects at the neuromuscular junction [1]. Since more than ten cases of MFS following COVID-19 infection have been reported to this date, and in a few cases antiganglioside antibodies were detected, there is evidence to support an aberrant immune-mediated response as the most likely pathogenesis [8]. The currently reported patient developed symptoms 18 days after the first dose of COVID19 vaccine. Post vaccination, MFS has been previously described in patients who received seasonal influenza vaccination, vaccine against H1N1, and after combined pertussis, diphtheria, and tetanus toxoid vaccine [9]. The most common side effects after vaccines against SARS-CoV-2 have been facial palsy and GBS [10]. Waheed et al. described a case of GBS after receiving the first dose of Pfizer COVID19 vaccine, which responded well to treatment with IVIG, similarly as in our patient [3]. The prognosis of Miller Fisher syndrome is generally favorable as it is mostly a self-limited disorder with IVIG leading to a more rapid recovery [11].

In conclusion, vigilance is needed for occurrence of any neurological complications related to vaccination with COVID-19 vaccines. Patients with acute ophthalmoplegia occurring after COVID-19 vaccination should be screened for the presence of anti-GQ1b antibody. If the antibody is present, intravenous immunoglobulin should be administered as it may hasten clinical improvement. Although case reports of suspected side effects of COVID-19 vaccines are of great importance for detection of rare adverse events in the post-marketing setting, vaccination should still be advocated as the benefit-risk ratio remains positive especially when compared to the substantially increased risk of serious adverse events after SARS-CoV-2 infection [12].

Supplementary Information The online version contains supplementary material available at https://doi.org/10.1007/s10072-021-05776-0.

\section{Declarations}

Ethical approval None.

Informed consent Informed consent was provided from the patient.

Conflict of interest None.

\section{References}

1. Lo YL (2007) Clinical and immunological spectrum of the Miller Fisher syndrome. Muscle Nerve. 36(5):615-27

2. Li Z, Li X, Shen J, Chan MTV, Wu WKK (2021) Miller Fisher syndrome associated with COVID-19: an up-to-date systematic review. Environ Sci Pollut Res Int. 28(17):20939-20944

3. Waheed S, Bayas A, Hindi F, Rizvi Z, Espinosa PS (2021) Neurological Complications of COVID-19: Guillain-Barre Syndrome Following Pfizer COVID-19 Vaccine. Cureus 13(2):e13426

4. European Medicines Agency. https://www.ema.europa.eu/en/ documents/product-information/comirnaty-epar-product-infor mation_en.pdf. Accessed on 20 Sep 2021)

5. Lee SH, Lim GH, Kim JS, Oh SY, Kim JK, Cha JK, Yun CH, Kang JK, Lee H, Song HK, Chung KC (2008) Acute ophthalmoplegia 
(without ataxia) associated with anti-GQ1b antibody. Neurology. 71(6):426-9

6. Odaka M, Yuki N, Hirata K (2001) Anti-GQ1b IgG antibody syndrome: clinical and immunological range. J Neurol Neurosurg Psychiatry. 70(1):50-5

7. Tatsumoto M, Odaka M, Hirata K, Yuki N (2006) Isolated abducens nerve palsy as a regional variant of Guillain-Barré syndrome. J Neurol Sci. 243(1-2):35-8

8. Biswas S, Ghosh R, Mandal A et al (2021) COVID-19 induced miller fisher syndrome presenting with autonomic dysfunction: a unique case report and review of literature. Neurohospitalist. $17: 1-6$

9. Krämer HH, Niemöller U, Döring K, Fockenberg S, Kaps M, Nedelmann M (2013) Postvaccination Miller Fisher syndrome after combined pertussis, diphtheria and tetanus toxoid vaccine. J Infect. 66(5):460-1
10. Goss AL, Samudralwar RD, Das RR, Nath A (2021) ANA investigates: neurological complications of COVID-19 vaccines. Ann Neurol. 89(5):856-857

11. Teener JW (2012) Miller Fisher's syndrome. Semin Neurol. 32(5):512-6

12. Barda N, Dagan N, Ben-Shlomo Y et al (2021) Safety of the BNT162b2 mRNA Covid-19 vaccine in a nationwide setting. N Engl J Med. 385(12):1078-1090

Publisher's note Springer Nature remains neutral with regard to jurisdictional claims in published maps and institutional affiliations. 